\title{
A new object captures attention-but only when you know which objects are old
}

\author{
Fook K. Chua
}

Published online: 31 December 2010

(C) Psychonomic Society, Inc. 2010

\begin{abstract}
According to the new object hypothesis (see, e.g., Yantis \& Hillstrom, Journal of Experimental Psychology: Human Perception \& Performance, 20, 95-107, 1994), an object appearing as a sudden onset captures attention because its appearance demands an immediate updating of visual short-term memory. This hypothesis was tested in three experiments using a procedure that allowed an object to be added to the display but, crucially, without incurring onset transients (Franconeri, Hollingworth, \& Simons, Psychological Science, 16, 275-281, 2005). The latter showed that an object inserted in this fashion failed to capture attention. As a test of the new object hypothesis, this procedure assumes that the observers had encoded the display before the new object was introduced. If this assumption is not fulfilled, the new and the old objects cannot be distinguished one from the other. It was, however, unclear whether the encoding had taken place in the Franconeri et al. experiments. We showed that when circumstances were congenial to the encoding of the display before an additional object interposed, then the object successfully captured attention. But when the encoding of the initial display was either difficult or impossible, the additional object failed to capture attention.
\end{abstract}

Keywords Attention - Attention capture - Correspondence problem . Visual short term memory $\cdot$ Object

The visual environment generally contains too much stimulation for us to apprehend all at once. To achieve some coherence in understanding the visual world, specific parts of the environment are selected, and attention is deployed accordingly. This is the issue of attentional control, and

F. K. Chua $(\bowtie)$

Department of Psychology, National University of Singapore,

Kent Ridge Crescent, Singapore 119260

e-mail: fkchua@nus.edu.sg research in this area has identified two modes of control. In the case of endogenous attention, the specific goals entertained by the observer determine the deployment of attention. In contrast, when attentional control is exogenous, features of the environment determine attentional deployment. In this case, because the observer's intentions are irrelevant, we say that attention is "captured." To understand exogenous control, researchers sought to discover those aspects of the visual environment that enable attentional capture.

An object that appears suddenly in a previously empty location (i.e., an abrupt onset) captures attention (Jonides \& Yantis, 1988; Müller \& Rabbitt, 1989; Theeuwes, 2004; Yantis \& Jonides, 1990; for reviews, see Yantis, 1998, 2000). Because an abrupt onset is accompanied by sudden changes to the local luminance profile at the onset's site, one question is whether the capture could be due to this low-level luminance change. It has been shown, for example, that sudden luminance transients elicit reflexive orienting (Breitmeyer \& Ganz, 1976; Breitmeyer \& Julesz, 1975; Nakayama \& Mackeben, 1989). The magnocellular pathway, which has been implicated in processing motion and in other forms of dynamic information, could be involved here. To rule out the onset transients account, Yantis and Hillstrom (1994) eliminated the luminance change accompanying the introduction of an object and reported that the new object did not, consequently, lose its capacity in capturing attention. Gellatly, Cole, and Blurton (1999) controlled for local luminance change and concluded that onset transients may not be necessary for capture to occur.

To explain how attentional capture may obtain even when the transients are obscured, Yantis and his colleagues proposed the new object hypothesis, which claims that an onset object captures attention in virtue of its novelty. The advent of the object brings with it a change to the visual environment, which compels an immediate updating of 
visual short-term memory (VSTM). To achieve this, an attentional interrupt is triggered, enabling the representation of the new object to be lodged in VSTM. In this way, the new object is attentionally prioritized and thus "captures" attention. There have been some novel demonstrations of the new object hypothesis (e.g., Rauschenberger, 2003).

The difficulties of controlling low-level luminance changes are well exemplified in Gellatly et al. (1999). Franconeri, Hollingworth, and Simons (2005) introduced a novel procedure for testing the new object hypothesis that cleverly circumvented the need to control for these low-level luminance effects. Observers first saw a display containing several objects. Midway during the trial, when these objects were momentarily ( $\sim 10 \mathrm{~ms}$ ) hidden from view, an additional object was added to the display. But as this object was introduced offstage, as it were, the low-level luminance transients that accompanied its appearance were invisible. The objects then reappeared, now as letters of the search array. Franconeri et al.'s results showed that a new object inserted in this fashion failed to capture attention. But when the onset transients of the additional object were visible, capture was observed. Chua (2009) replicated these results.

These findings contradicted the new object hypothesis because they suggested that the transients that accompanied an onset were necessary in ensuring attentional capture success. The Franconeri et al. (2005) displays were austere, containing three or four or five identical objects ("digital" figure 8, Todd \& van Gelder, 1979), each occupying a unique location. Since observers were aware that search would be conducted among these objects when they were transformed into letters, it seems reasonable for one to assume that attention would be deployed on the objects. One of the insights of the change blindness literature is that attention itself may sometimes not be sufficient for detection of change (Simons \& Levin, 1997). What is important is that, before the change, the to-be-changed object must be encoded so that a comparison may be made after the change is wrought.

Consider a display that contains an initial set of identical objects. Another identical object is then added to the display. When this new object's onset transients are apparent, the visual system can use them to flag its location. But, if these transients are invisible, how might the system discriminate the new object from the initial set? The task facing the visual system is the correspondence problem (Pylyshyn, 1989, 2004). Encodings of the display before the additional object is inserted have to be compared with the encodings after the insertion is made. The new object is the entity for which no correspondence can be established. Yantis and Gibson (1994) made a similar argument: An object is new if the visual system is unable to establish spatiotemporal continuity between the object and the representation previously stored in VSTM.
Regarding the Franconeri et al. (2005) procedure, the question is whether there was encoding of the display before the objects were concealed so that correspondence could be established with the items as they emerged from concealment. The search task did not require explicit encoding of the display; neither was there evidence that the procedure promoted any implicit encoding. Furthermore, the display included an annulus that contracted as the trial progressed. Moving objects capture attention (Abrams \& Christ, 2003; Franconeri \& Simons, 2003). Thus, even if observers had encoded the objects at the start of the trial, attention could have switched to the annulus once it began contracting. The initial encoding of the objects is likely to be volatile (Becker \& Pashler, 2002; Rensink, 2000), and if not maintained, will disintegrate (e.g., inattentional amnesia, Wolfe, 1999). If observers switched attention to the annulus and therefore were not able to maintain the initial encoding of the objects, there would have been no internal representation of them when the search array was revealed. Therefore, no correspondence between the letters of the search array and the original set of objects can be made. Consequently, the additional object cannot be distinguished from the old objects. If the visual system is blind to which among the objects is new, it is not surprising that the system is unable to prioritize that object.

Davoli, Suszko, and Abrams (2007) argued that the Franconeri et al. (2005) results might have arisen because the moving annulus had captured attention. To test this hypothesis, they used a variation of the Franconeri et al. paradigm in which a static mask was pitted against a moving mask. They showed that although the additional object failed to capture attention when the mask was moving, capture succeeded when the mask was static. Hollingworth, Simons, and Franconeri (2010), reviewing the Davoli et al. experiments, commented that the latter results might have been due to the particular instructions administered to the observers (Hollingworth et al., 2010, p. 1301). It is unclear, however, whether the instructions per se were critical, since Hollingworth et al. failed to replicate the Davoli et al. results, even when they administered the same Davoli et al. instructions to their observers (Hollingworth et al., 2010, Experiment 1).

On the assumption that the objects had been implicitly encoded, the failure to witness capture would constitute evidence against the new object hypothesis. But, if there had been no encoding in the first place, the new object hypothesis could not be deemed to have been adequately tested. The goal of the present set of experiments was to explore how encoding of the initial set of ("old") objects could facilitate the discrimination of the new from the old objects. In the experiments (except Experiment 2c), the observers were neither instructed explicitly on how to encode the objects, nor were they oriented to the additional 
object that could be inserted, in the display. They were merely told that their task was to search for one of two target letters in the search array. Instead, implicit encoding of the display was facilitated in two ways. In Experiment 1, the perceptual load was reduced, which should have facilitated encoding of the display. In Experiment 2, the Franconeri et al. (2005) procedure was varied by allowing the objects to move while keeping the annulus stationary. The intention here was to coax observers to track the moving objects, thereby ensuring that their representations would be updated continuously. To anticipate the results, this manipulation in Experiment 2 resulted in attentional capture. If capture success hinges on a veridical representation of the initial display, undermining this encoding should affect adversely the new object's capacity in capturing attention. In Experiment 3, the set size was increased considerably so that observers would not be able to simultaneously track all the objects. This meant that the encoding of the objects, before they disappeared from view, would not be complete.

\section{Experiment 1}

The task required the observer to perform a visual search on an array of letters. Since the objects' locations were completely irrelevant to the task, it was unlikely that the observers would explicitly encode information about the objects' locations. The question was whether they might be encoded implicitly. In the Franconeri et al. (2005) experiments, the display at the start of the trial consisted only of three or four or five identical objects. This load is probably within the capacity of VSTM, which has been estimated to be around four objects (e.g., Cowan, 2001; Todd \& Marois, 2004). The display, however, also included an annulus, which contracted as the trial progressed, that was likely to add to the overall perceptual load. But whether the total load would then exceed VSTM capacity is unclear. In Experiment 1, the perceptual load was reduced in a bid to facilitate the full encoding of the display.

\section{Experiment 1}

Method

Participants There were 21 observers recruited from the subject participant pool who participated for course credit. All observers had normal or corrected-to-normal vision.

Procedure First, a description of how a typical trial progressed will be provided. Observers initially saw several placeholders (a "digital" figure 8, Todd \& van Gelder,
1979), located on the circumference of an invisible circle. The diameter of the circle subtended a visual angle of $12^{\circ}$ with the observers seated about $50 \mathrm{~cm}$ from the display. The placeholders' positions were randomly selected from 12 possible locations, subject to the constraint that there would be empty space between at least some adjacent pairs. The placeholders were black in color $\left(30 \mathrm{~cd} / \mathrm{m}^{2}\right)$, and the background was gray $\left(60 \mathrm{~cd} / \mathrm{m}^{2}\right)$. Each placeholder subtended a visual angle of $0.7^{\circ}$ (height) $\times 0.5^{\circ}$ (width). These objects, which were stationary throughout the trial, were surrounded by an annulus formed by two concentric circles, the edges of which were 2-pixel wide and drawn in black, matching the luminance of the placeholders (Fig. 1a). The width of the annulus subtended an angle of $1^{\circ}$. The diameter of the outer ring subtended an angle of $18.5^{\circ}$. The first frame was presented for $300 \mathrm{~ms}$, after which the annulus started contracting (Fig. 1b). Midway during the trial, the contracting annulus passed over the stationary placeholders, obscuring them completely for $10 \mathrm{~ms}$ (Fig. 1c). While they were completely hidden, the placeholders shed several lines and were transformed into letters. On half of the trials, an additional letter was added at this stage. But, because the addition was made when the annulus blocked the objects from view, the onset transients (and also the offset transients when the placeholders shed line segments) were not visible. As the annulus contracted further, the letters now reappeared from under the annulus (Fig. 1d, e). The annulus stopped contracting when it subtended an angle of $4.6^{\circ}$, at which point the letters surrounded the annulus (Fig. 1f). The entire animation sequence, which contained 18 frames, took $180 \mathrm{~ms}$. The search letters remained in view for a further $100 \mathrm{~ms}$, after which they disappeared.

There were three main variables: (a) number of objects in the display (set size variable), (b) whether an object was added to the display (additional object variable), and (c) for the additional object trials, whether the additional object was a target or a distractor (additional object $=$ target vs. additional object $=$ distractor ). At the start of the trial, there were two or three or four objects. For the no additional object (baseline) condition (50\% of trials), the search set size at the end of the trial remained the same. For the additional object condition ( $50 \%$ of trials), at the end of the trial, the search set size was increased by 1 , to three or four or five objects (letters). For these trials, the additional object could either be a target or a distractor letter. The 1/d design was implemented so that the additional object was the target on only $1 / d$ (where $d$ is the set size) of the trials. For example, when the set size was four, the target was the additional object on only $25 \%$ of the trials. There were two possible target letters, E or $\mathrm{H}$. The distractors were randomly selected, without replacement, from the letters A, C, U, P, and S. 
Fig. 1 The sequence events in a typical trial in Experiment 1. a At the start of the experiment, the annulus surrounded the stationary placeholders. b As the trial progressed, the annulus shrank, and $\mathbf{c}$ blocked the objects from view. d, e When the annulus shrank further, the objects, now transformed into letters, were revealed. On some trials, an object (the letter "E" at about the 12 o'clock position) was added to the display when the placeholders were hidden from view. $\mathbf{f}$ The annulus stopped shrinking at the end of the trial and was surrounded by letters (a)

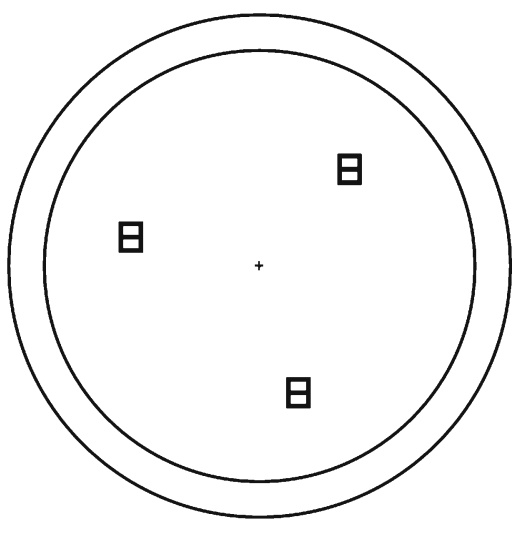

(b)
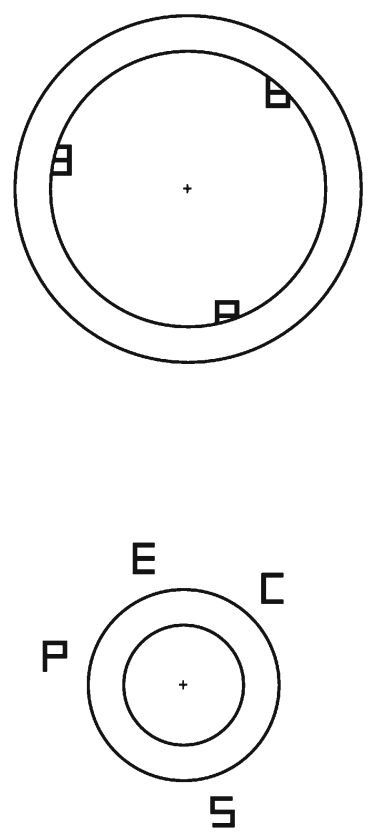

(e) (d)

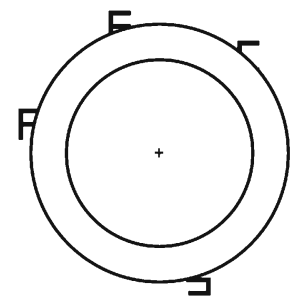

(c)

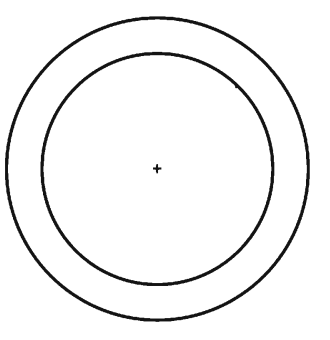

For each block, there were 48 trials: 24 trials each for the two targets. For each of these 24 trials, there were 12 no additional object and 12 additional object trials. These 12 trials were divided into three or four or five trials for the three different set size conditions. Thus, for each block of trials, there were only six trials $(12.5 \%)$ in which the new object was the target, making them quite infrequent. The task was described to the observers as a visual search task. Observers were briefed to be fast but accurate in identifying which of two possible target letters appeared in the search array. No other instructions were imparted. Feedback (a beep sound) was provided when an error was committed. The error trial was rerun toward the end of the block. There were, altogether, 11 blocks of trials. The data from the first block, which was regarded as a practice block, were not analyzed.

\section{Results and discussion}

The latency and accuracy data for the additional object conditions, as a function of set size, are presented in Fig. 2. The main dependent variable was reaction time (RT). The accuracy data were first analyzed to ascertain that speedaccuracy trade-off was not an issue. Accuracy for the additional object $=$ target condition was lower than that for the additional object $=$ distractor condition. The mean latencies of the former condition were, however, longer, suggesting that speed-accuracy trade-off was not an issue. A formal analysis of the accuracy data revealed no effects whatsoever. ${ }^{1}$

For the main analysis based on the latency data, a linear regression was computed for each of the additional object conditions. The slopes from the regression analyses were then entered as the dependent variables in the ANOVA. The diagnostic for capture by a new object is a flat search function for the additional object $=$ target condition, but is a linearly increasing function for the additional object $=$ distractor condition. First, the slopes for the baseline (no additional object) and the two additional-object conditions were submitted to a one-way ANOVA. The main effect was significant, $F(2,40)=5.880, p<.01, \eta_{\mathrm{p}}^{2}=0.23$. Further analysis revealed that the slopes of the baseline (slope $=31.2)$ and the additional object $=$ distractor $($ slope $=38.1)$ conditions were comparable, $F(1,20)=1.96, p<.2, \eta_{\mathrm{p}}^{2}=0.09$. But the slopes of the additional object $=$ distractor and additional object $=$ target conditions (slope $=16.6$ for the latter condition) were significantly different, $F(1,20)=6.414$,

\footnotetext{
${ }^{1}$ A 2 (additional object) $\times 3$ (set size) ANOVA with accuracy as the dependent variable revealed no significant effects, $F(1,20)=3.842$, and $F(2,40)=2.123, p>.05$, for the additional object and set size variables, respectively. The interaction effect was also not reliable, $F<1$.
} 


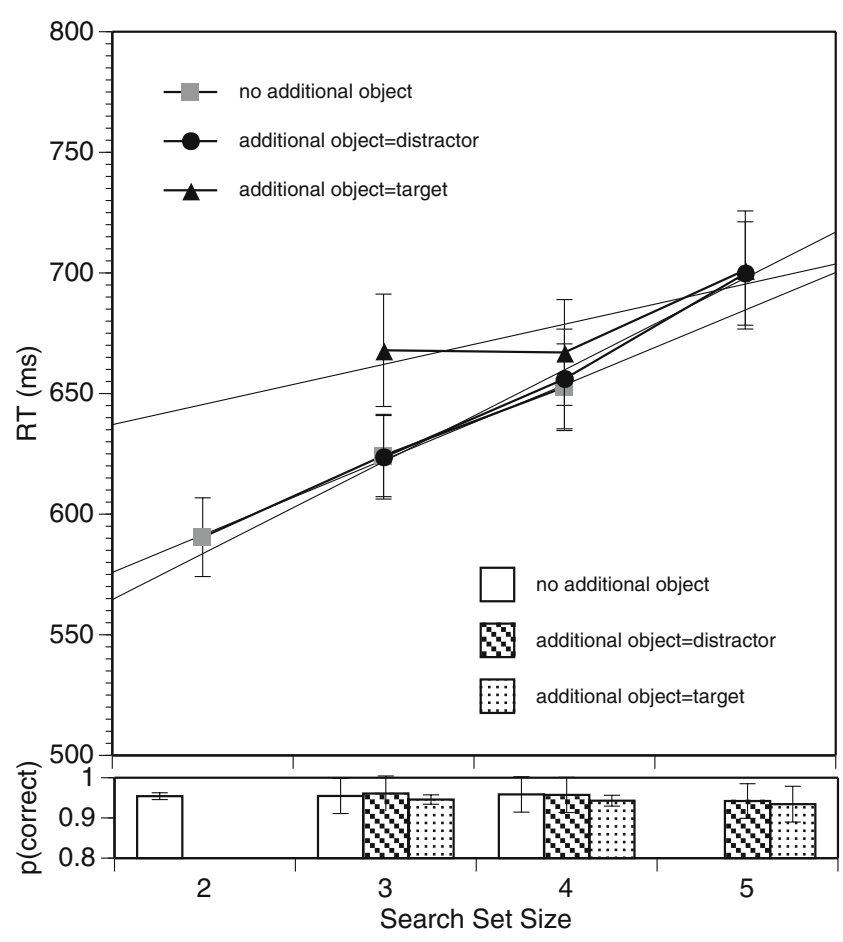

Fig. 2 Mean reaction times of the three additional object conditions as a function of search set size. Also drawn on the plot are the best fitting linear regression functions for the conditions. Error bars $=1 S E$ $p<.05, \eta_{\mathrm{p}}^{2}=0.24 .^{2}$ In Franconeri et al. (2005) and Chua (2009), the slopes of these two conditions were comparable.

The slope of the additional object $=$ target function was significantly shallower than that of the additional object $=$ distractor function, but the function was not flat. A one-way ANOVA of the three set sizes, with RT as the dependent variable, revealed a significant set size effect, $F(2,40)=3.814$, $p<.05, \eta_{\mathrm{p}}^{2}=0.16$. These data therefore failed the diagnostic for attentional capture. Further scrutiny of the data showed that RTs were not statistically different for set sizes 3 and 4 $(F<1)$. One plausible interpretation is that when load was low (two or three objects), the encoding of the placeholders could be maintained even in the face of a competing moving object (the annulus). But when the load increased to four objects, a veridical representation of the placeholders could not be maintained. This interpretation, however, is complicated by the fact that, for set size 4, the RT for the additional object $=$ target condition was not statistically different from the RTs for the other two conditions $(F<1)$. Hollingworth et al. (2010), who used the same set sizes as in this experiment, reported comparable slopes for the two additional-object conditions. On balance, then, the results do not show that the additional object captured attention. But, the question still

\footnotetext{
${ }^{2}$ The results from the analysis of latency data converged nicely with the slopes analysis. A 2 (additional object) x 3 (set size) ANOVA showed main effects of the additional object and of set size variables, $F(1,20)=8.338$, $p<.001, \eta_{\mathrm{p}}^{2}=0.29$, and $F(2,40)=23.575, p<.001, \eta_{\mathrm{p}}^{2}=0.54$, respectively, and a marginally significant interaction effect, $F(2,40)=$ $3.215, p=.051, \eta_{\mathrm{p}}^{2}=0.14$.
}

remains whether their observers had encoded the initial display. Since one can only go so far in manipulating perceptual load in this paradigm, in the next two experiments, we used a different strategy to coax observers to maintain their attention on the objects.

\section{Experiment 2}

In Experiment 1, the objects remained stationary while the annulus contracted. Since observers knew that the placeholders would turn into letters as the trial progressed, it was likely that attention would be deployed to the placeholders initially. But, as the trial began, the annulus started contracting. Because the annulus was the only object that was changing, it was probable that attention would switch to the annulus rather than to the search objects (Abrams \& Christ, 2003; Franconeri \& Simons, 2003). The competition for attentional resources from the contracting annulus may explain why encoding of the objects could not be maintained, even when perceptual load was reduced (see Davoli et al., 2007, for a similar argument). If this conjecture is correct, moving the objects, but keeping the annulus stationary, could succeed in keeping attention rooted to the placeholders throughout the trial.

In Experiment 2, the placeholders moved across the display while the annulus remained stationary. The goal was to maintain the observers' attention on the placeholders right up to the point that they were hidden from view. Tracking the placeholders would mean continuously updating their location information in VSTM. The additional object would stand out as the only object for which no previous record exists. Insofar as VSTM has to be immediately updated with the representation of this object, we should witness attentional capture. Three experiments (Experiments 2a, 2b, and 2c) were conducted, all using the same procedure. Experiment $2 \mathrm{a}$ is described first. An aspect of its results complicated their interpretation. Experiments $2 b$ and $2 \mathrm{c}$ were conducted to resolve this problem.

Experiment 2a

\section{Method}

Participants The observers were 16 new students recruited from the same subject pool. They participated under the same motivational condition as Experiment 1.

Procedure The design of Experiment 2a was identical to that of Experiment 1, except that the search set size was four or five or six letters. The stationary annulus subtended a visual angle of approximately $12^{\circ}$. The sequence of events is depicted in Fig. $3(\mathrm{a}-\mathrm{f})$. When the trial began 
Fig. 3 The sequence of events in Experiment 2. a The location of the placeholders and the annulus at the start of the trial. b The placeholders started moving outward in a linear trajectory. c The placeholders were completely obscured by the annulus halfway through the trial; at this stage, the placeholders shed the lines camouflaging the letters. For the additional object trial, a new letter was added at this point. d The letters emerged from the annulus; the additional object (at the 12 o'clock position) was revealed. e The placeholders continued their linear trajectory after they emerged, and then f halted (a)
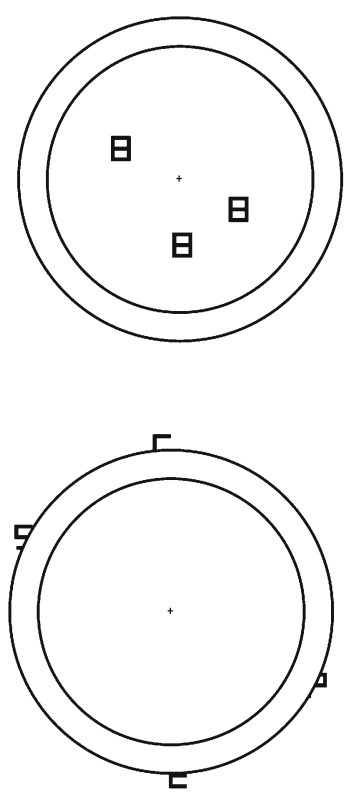

(d) (b)
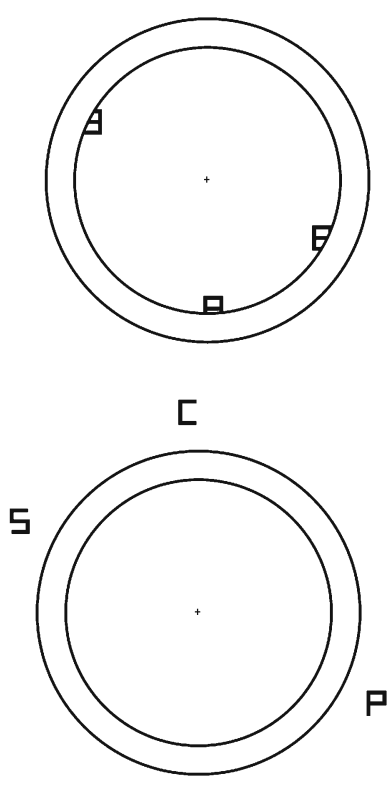

E

(e) (c)

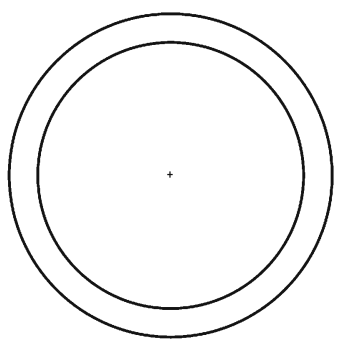

ᄃ

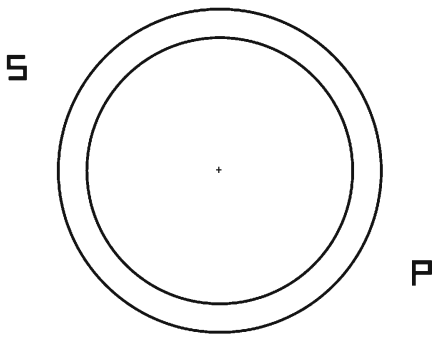

E
(Fig. 3a), the three or four or five placeholders were located on the circumference of an invisible circle (visual angle = $5^{\circ}$ ), surrounded by the annulus. As the trial progressed, the placeholders moved in a straight-line trajectory toward the boundary of the display (Fig. 3b). Midway during the trial (90 ms after they began to move), they passed under the stationary annulus and, for $10 \mathrm{~ms}$, were completely obscured (Fig. 3c). The old items shed the line segments and turned into letters. On half of the trials, an additional object was inserted to the display at this juncture, but, since this occurred when the objects were hidden, its onset transients were invisible. The items (now letters) then moved out from under the annulus (Fig. 3d), continuing along their linear trajectories (Fig. 3e). They traveled for a further $90 \mathrm{~ms}$ and then halted (Fig. 3f).

\section{Results}

This section is organized as follows. First, the results of Experiment $2 \mathrm{a}$ are discussed. To anticipate the results, the intercept of the additional object $=$ target condition was found to be significantly higher than that of the other two conditions, which complicated the interpretation of the data. Consequently, two additional experiments (Experiments $2 \mathrm{~b}$ and 2c) were conducted to resolve the intercept issue.

The data of Experiment $2 \mathrm{a}$ were treated in the same manner as described earlier. The mean RTs and accuracy rates of the baseline and the two additional object conditions as a function of set size are presented in Fig. 4 (left panel). Again, the accuracy data were first analyzed. A 2 (additional object) $\times 3$ (set size) ANOVA revealed a set size effect, $F(2,30)=8.480, p<.001, \eta_{\mathrm{p}}^{2}=0.36$. The additional object main effect and the interaction effect were not reliable $\left(F_{\mathrm{S}}<1\right)$. As in Experiment 1, speed-accuracy tradeoff was not an issue here.

A one-way ANOVA of the slopes of the three conditions revealed a significant effect, $F(2,30)=8.282, p<.001$, $\eta_{\mathrm{p}}^{2}=0.36$. Further unpacking the main effect showed that the difference between the baseline (slope $=43.7, r^{2}=1.0$ ) and the additional object $=$ distractor $\left(\right.$ slope $\left.=35.3, r^{2}=.99\right)$ was not significant, $F(1,15)=1.746, p>.2, \eta_{\mathrm{p}}^{2}=0.10$. However, the two additional object conditions had reliably different slopes, $F(1,15)=8.242, p<.05, \eta_{\mathrm{p}}^{2}=0.35$. Analyses of the RT data were consistent with the slope analysis. $^{3}$ The slope for the additional object $=$ target condition was $8.84\left(r^{2}=.93\right)$. But a one-way ANOVA of the RTs revealed that the function was essentially flat, $F<1$. Furthermore, the intercept of the additional object $=$ target condition also differed substantially from the other two functions. A one-way ANOVA of the three intercepts revealed a significant effect, $F(2,30)=12.981, p<.001$,

\footnotetext{
${ }^{3}$ A 2 (additional object conditions) x 3 (set size) ANOVA using latencies as the dependent variable revealed that the main effects were significant, $F(1,15)=23.373, p<.001, \eta_{\mathrm{p}}^{2}=0.61, F(2,30)=10.287$, $p<.001, \eta_{\mathrm{p}}^{2}=0.41$, for the additional object and set size conditions, respectively. The interaction effect was also significant, $F(2,30)=$ 5.123, $p<.02, \eta_{\mathrm{p}}^{2}=0.25$.
} 


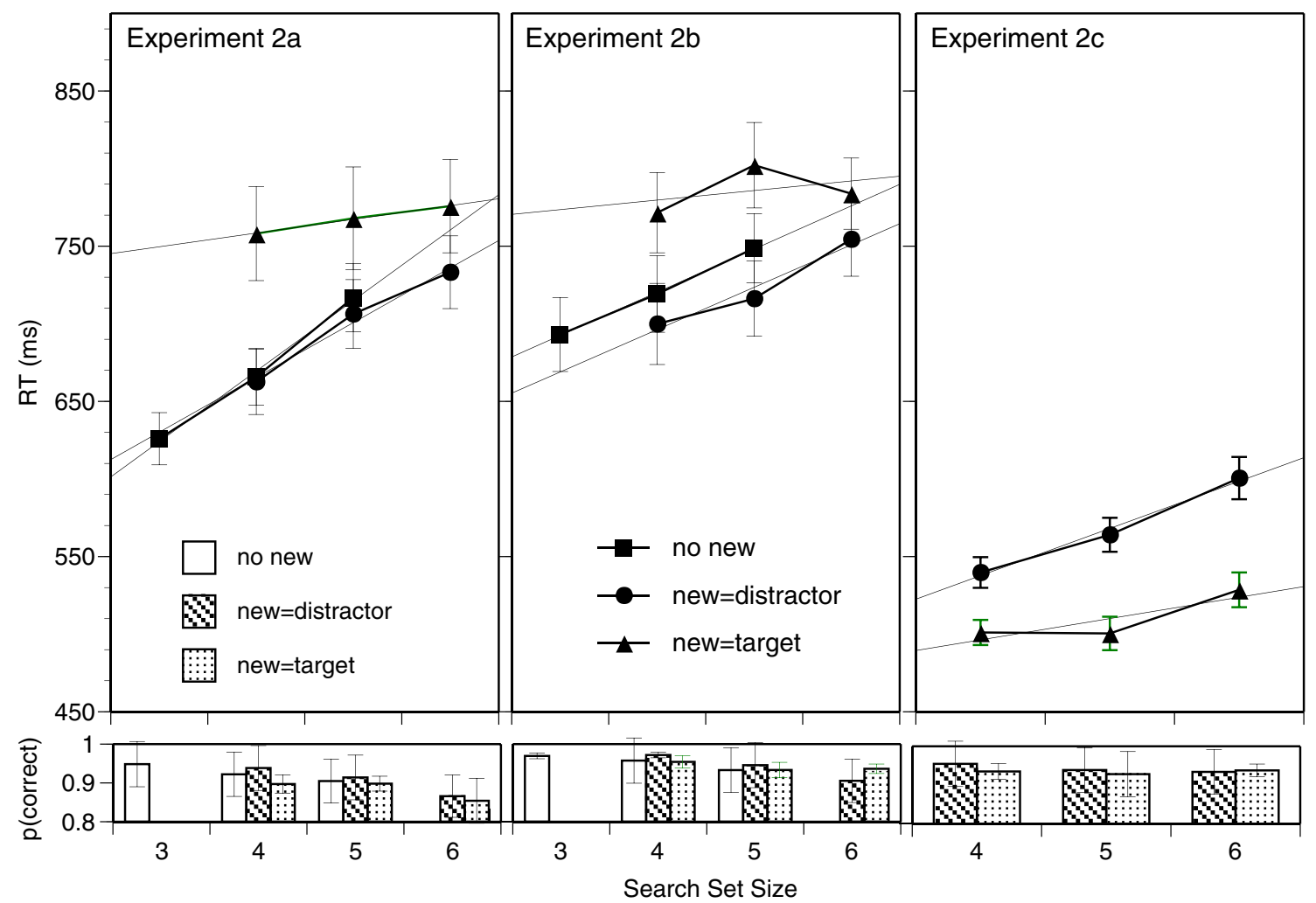

Fig. 4 c Mean RTs of the additional object conditions as a function of search set size for Experiments 2a, 2b, and 2c. Also drawn on the plots are the best fitting linear regression functions for the conditions. Error bars $=1 S E$

$\eta_{\mathrm{p}}^{2}=0.46$. An additional analysis revealed that there was no difference between the baseline (intercept $=493 \mathrm{~ms}$ ) and the additional object $=$ distractor $($ intercept $=523 \mathrm{~ms}$ ) functions, $F<1$. But the additional object $=$ target condition (intercept $=$ $721 \mathrm{~ms}$ ) had a substantially higher intercept than did the additional object $=$ distractor condition, $F(1,15)=14.323$, $p<.001, \eta_{\mathrm{p}}^{2}=0.49$.

The interpretation of the flat search function of the additional object $=$ target condition as evidence for capture is complicated by the finding that its intercept is substantially higher than the additional object $=$ distractor condition. ${ }^{4}$ If the additional object had been prioritized because it was new, and attention was deployed first to its location, the mean latency ought to have been shortest when the additional object was a target. Contrary to this prediction, the mean RTs of the various set sizes for the

\footnotetext{
${ }^{4}$ Finding an overall set size effect for the accuracy data but not an interaction effect between set size and the additional-object conditions posed another problem to the capture interpretation. Further analyses of the accuracy data showed that the set size effect was due largely to the additional object $=$ distractor condition, $F(2,30)=9.577, p<.001$, $\eta_{\mathrm{p}}^{2}=0.49$. For the additional object $=$ target condition, the accuracy rates of the three set sizes were not reliably different, $F(2,30)=2.932$, $p>.07, \eta_{\mathrm{p}}^{2}=0.16$.
}

additional object $=$ target condition were longer than the additional object $=$ distractor condition. The observation of a higher intercept was also reported by Chua (2009, Experiment 1b), even when the onset transients of the additional object were visible (see Fig. 2, center).

\section{Experiment $2 b$}

Because the results observed in Experiment 2a appear important to the attentional capture debate, we decided to replicate, with some modifications, the experiment $(n=16)$. The identification of the letters, as they emerged from under the annulus, may have been affected by the outer edge of the annulus, which had the same luminance as the letters (e.g., contour interactions). In the replication, the body of the annulus had a luminance that was $62 \mathrm{~cd} / \mathrm{m}^{2}$ (recall that the background was $60 \mathrm{~cd} / \mathrm{m}^{2}$ ) so that the outer circumference of the annulus was a luminance edge. Another modification was that the objects were first located outside the annulus and, as the trial progressed, they traveled toward the center of the display. As in Experiment 2a, observers were told to search the letter array for the targets. No other instructions were conveyed. The accuracy and RT data are presented in Fig. 4 (center panel). The results of the replication were, in 
essence, identical to those of Experiment 2a. ${ }^{5}$ Of particular interest, the substantially higher intercept for the additional object $=$ target condition was replicated.

\section{Experiment 2c}

In Chua (2009, Experiment 3c), the intercept of the additional object $=$ target function was not significantly different from the baseline condition, which contrasted with his results for Experiments 2 and $3 \mathrm{a}$ in the same report. One difference between the procedures in these two groups of experiments is that in Chua's Experiment $3 \mathrm{c}$, the observers were told explicitly to attend to the additional object, whereas the observers in his Experiments 2 and 3a were not forewarned that an additional object would appear in $50 \%$ of the trials. In Franconeri et al. (2005), Davoli et al. (2007), and Hollingworth et al., (2010), the observers were all alerted to the additional object, and their data all showed that the intercepts of the additional object functions were equivalent. Chua (2009) argued that postidentification checking may explain the higher intercept in his experiments. The hypothesis tested in our present Experiment $2 \mathrm{c}$ is that the postidentification checking processes may be eliminated by forewarning observers that an object will be added to the display.

For Experiment 2c $(n=16)$, a 2 (additional object: distractor vs. target) $\times 3$ (search set size $=$ four, five, or six objects) factorial design was used. The procedure was identical to that in Experiment 2b. Observers were told, via a video clip, that an additional object would be added to the display when the placeholders moved under the stationary annulus. As in Hollingworth et al.'s instructions (2010, Experiment 1B), observers were told that more often than not, the additional object will not be one of the two target letters. To underscore these instructions, observers then went through several demonstration trials in which the program ensured that the additional object was a distractor letter.

The accuracy and RT data are presented in Fig. 4 (right panel). Analyses of the accuracy data in a 2 (additional

\footnotetext{
${ }^{5}$ The same analyses as in the main experiment. First, analysis of the accuracy data showed that speed-accuracy trade-off was not an issue. In the main analysis, the slopes of the three conditions $\left(32.2, r^{2}=1.0\right.$; $33.0, r^{2}=.99$; and $4.8, r^{2}=.26$; for the baseline, additional object $=$ distractor, and additional object $=$ target conditions, respectively) were compared in a one-way ANOVA. There was a significant slope effect, $F(2,30)=7.356, p<.005, \eta_{\mathrm{p}}^{2}=0.33$. Further analysis showed that although there was no difference between the slopes of the baseline and the additional object $=$ distractor conditions, $F<1$, the slopes of the two additional object conditions were significantly different, $F(1,15)=8.269, p<.05, \eta_{\mathrm{p}}^{2}=0.36$. A one-way ANOVA of the mean latencies $(785,804$, and $794 \mathrm{~ms}$, for the set size 4,5 , or 6 conditions, respectively) of the additional object $=$ target condition revealed no effect, $F<1$. But, as in Experiment 2a, the intercept for the additional object $=$ target condition was significantly higher than that for the additional object $=$ distractor condition $(775$ vs. $589 \mathrm{~ms}$, respectively), $F(1,15)=16.711, p<.001, \eta_{\mathrm{p}}^{2}=0.61$.
}

object: target vs. distractor) $\times 3$ (set size) ANOVA revealed no interesting effects: For the condition variable, $F<1$; for the set size variable, $F(2,30)=1.48, p>.2, \eta_{\mathrm{p}}^{2}=0.08$; for the interaction effect, $F(2,30)=1.308, p>.2, \eta_{\mathrm{p}}^{2}=0.07$. The main analysis showed that the slope $\left(M=30.41, r^{2}=.99\right)$ of the additional object $=$ distractor condition is significantly steeper than the slope $\left(M=13.7, r^{2}=.73\right)$ of the additional object $=$ target condition, $F(1,15)=14.306, p<.002$, $\eta_{\mathrm{p}}^{2}=0.49$. The latter slope is not flat: A one-way ANOVA of the RTs for the three set sizes $(M \mathrm{~s}=501,500$, and $528 \mathrm{~ms}$ for set sizes 4,5 , or 6 , respectively) revealed a significant effect, $F(1,15)=5.648, p<.01, \eta_{\mathrm{p}}^{2}=0.27$. A 2 (additional object $\times$ set size) ANOVA of the latency data revealed significant effects for following conditions - additional object, $F(1,15)=149.36, p<.001, \eta_{\mathrm{p}}^{2}=0.91$; set size, $F(2,30)=19.133, p<.001, \eta_{\mathrm{p}}^{2}=0.56$; and the interaction, $F(2,30)=7.959, p<.005, \eta_{\mathrm{p}}^{2}=0.36$. The disparity in latency between the two additional object conditions increased with set size. Finally, the two conditions had statistically similar intercepts ( $446 \mathrm{~ms}, S E=17.6$; vs. $455 \mathrm{~ms}$, $S E=18$, for the additional object $=$ distractor and additional object $=$ target conditions, respectively), $F<1$.

\section{Results and discussion}

The results of Experiment 2 may be summarized as follows. In Experiments $2 \mathrm{a}$ and $2 \mathrm{~b}$, the baseline and the additional object $=$ distractor conditions have slopes of comparable magnitude. In contrast, the slope of the additional object = target condition is essentially flat. In Experiment 2c, although the slope cannot be considered flat, it is significantly shallower than that in the additional object $=$ distractor condition. The results of Experiments $2 a$ and $2 b$ stand in nice contrast to those of Chua (2009, Experiment 1), in which the search functions of all three conditions had comparable slopes. The two sets of experiments were identical in all respects except that in Chua, the annulus contracted while the objects remained stationary, whereas in the present Experiment 2, the annulus remained stationary while the objects moved.

The flat search function for the additional object $=$ target condition in Experiments 2a and 2b implies that set size did not modulate search time, which may be interpreted as evidence of capture by the additional object. There is one complication to the interpretation: The latencies were longer in the additional object $=$ target condition than in the additional object $=$ distractor condition. If capture had occurred, and the object that captured attention was a target letter, search ought to have terminated immediately, with a shorter RT as the result. Chua (2009) also reported higher intercepts for the additional object $=$ target condition, even when the transients of the additional object were visible. In contrast, in a comparable experiment, Franconeri et al. 
(2005) reported that the intercepts for all the conditions were, more or less, the same. Chua (2009) argued that the higher intercept was perhaps mainly due to postidentification checking processes. In his experiments, the additional object was inserted on $50 \%$ of the trials. Because the $1 / \mathrm{d}$ design was also implemented, the additional object was also the target object only on about $10 \%$ of the trials. This meant that it would be quite rare for the attentional system to engage the target immediately on commencement of search. As a result, postidentification checking could have been triggered to confirm that the object was indeed the target.

One difference between Franconeri et al. (2007) and Chua (2009, all experiments except Experiment 3b) is that although the participants in Franconeri et al. (see also, Davoli et al., 2007; Hollingworth et al., 2010) were forewarned that an additional object would be inserted midway during the trial, the observers in Chua (and the observers in Experiments $2 \mathrm{a}$ and $2 \mathrm{~b}$, above) were not similarly forewarned. One possibility is that the expectation of the additional object could modulate the process of postidentification checking. To test this hypothesis, observers in Experiment 2c were told explicitly to expect the addition of an object midway through the trial. The results showed that, with forewarning, the intercepts in the two additional-object conditions were comparable. The expectation that an object would be added and having the expectation realized could well have suppressed postidentification checking. Although the slope of the additional object $=$ target function was not flat, it should be noted that, for the different set sizes, the latencies were significantly shorter in the additional object $=$ target condition than in the additional object $=$ distractor condition.

The results of Experiments $2 \mathrm{a}$ and $2 \mathrm{~b}$ are consistent with those of Davoli et al. (2007), in which a stationary mask was pitted against a moving mask. Capture by a new object was demonstrated only in the former condition, which led Davoli et al. to postulate that the moving mask could well have wrested attention away from the objects. ${ }^{6}$ The implication is that, for capture to occur, focusing attention on the objects and not on the mask was necessary.

In Experiment 2, the annulus was stationary, which must at least weaken, if not eliminate, it as a competitor for attention. As in Davoli et al. (2007), it is reasonable for one to assume that attention was focused on the objects, at least initially. The question is whether attending to the objects would be sufficient for capture success. For example, Simons and Levin (1997) argued that merely focusing attention on the to-be-changed object might not guarantee detecting change; encoding the to-be-changed object is crucial.

Chua (2009, Experiment 3) showed that when conditions were optimal for the implicit encoding of the old objects, or

\footnotetext{
${ }^{6}$ As noted earlier, Hollingworth et al. (2010) failed to replicate the results of Davoli et al. (2007).
}

when observers were explicitly instructed to attend to the additional object, the latter succeeded in capturing attention. Earlier, Theeuwes (1995) made essentially the same argument to explain the inconsistency between his results and those reported by Yantis and Hillstrom (1994). He observed that the Yantis and Hillstrom displays contained fewer objects that occupied locations that were also more constrained. His argument was that observers could have (implicitly) encoded the initial display. Thus, when an object was added, a comparison of what had been encoded and what was currently on the screen would automatically flag that object as the new entity. In Experiment 2, the objects also moved continuously, which meant that attention probably remained focused on them. Tracking the objects meant continuously updating their locations. Capture by the new object could be due not just to attention to, but also to the encoding of, the moving objects.

Pylyshyn's (1989) FINST framework provides a useful way of thinking about the processing that obtained. The relatively small set sizes permit separate indexes to be maintained for the objects, therefore allowing them to be tracked effectively. The important point here is that when objects are individuated, the additional object will stand out as the only item for which no index has been assigned. This will trigger an updating of VSTM, with attention immediately deployed to that object so that its aspects may be discovered. In effect, the new object captures attention.

If the identification of the additional object as a new entity is dependent on the system's capacity in individuating the initial set of objects, undermining this capacity will make discriminating the new object from the initial set more arduous. In Experiment 3, we tested this hypothesis by increasing the set size of the moving objects. According to estimates from MOT experiments, about four or five separate indexes can be maintained. If the number of moving objects exceeds the number of separate indexes that the visual system can maintain simultaneously, at least some objects in the set will not be tracked. Thus, these untracked objects cannot be distinguished from a new object added just before the objects turned into search letters. The prediction would be that once the system fails in encoding the entire set of old objects, the additional object will not stand out as a new entity and therefore attentional capture by this new object will not occur.

\section{Experiment 3}

In Experiment 3, the set sizes used were seven, eight, or nine items to ensure that simultaneous tracking of the entire set was likely to be unachievable. The objects moved in a predictable linear trajectory, which lended them to be easily grouped, with attention deployed to the group as a single entity. However, individuating the objects in the group 
would be difficult. The question was whether a new object, introduced without onset transients, would still be prioritized.

\section{Method}

Observers The observers were 17 new students recruited from the same subject pool as that of the other experiments.

Procedure The procedure was identical to that in Experiment 2a. There were two main changes to the design. First, the search set size was seven or eight or nine letters. Second, only the two additional object conditions were included (i.e., the baseline condition was eliminated) in order to keep the duration of the experiment to approximately 30 min. Each block contained 48 trials; half of these had the letter "E" as the target, and the other half had the letter "H." As in the previous experiments, $1 / \mathrm{d}$ design was again implemented. A 2 (additional object: distractor vs. target) $\times 3$ (search set size) factorial design was used. The procedure was identical to that in Experiment 2, except that the search array remained in view until the observer responded.

Each observer went through 10 blocks of trials and an additional $1 / 2$ block of practice trials, and the data from these were not analyzed.

\section{Results and discussion}

The mean RTs and accuracy rates for the additional object conditions as a function of search set size are displayed in Fig. 5. Analysis of the accuracy data showed that a speedaccuracy trade-off was not an issue for these data. A 2 (additional object) $\times 3$ (set size) ANOVA revealed that the only reliable effect was the set size effect, $F(2,32)=9.786$, $p<.001, \eta_{\mathrm{p}}^{2}=0.29$. All other effects were not significant.

The RT data were treated as described previously, and the slopes for the additional object $=$ distractor $(21 \mathrm{~ms}$, $\left.r^{2}=1.0\right)$ and additional object $=$ target conditions $(22 \mathrm{~ms}$, $\left.r^{2}=.97\right)$ were then subjected to a formal analysis. There was no difference between them, $F<1$. The intercepts for the two functions ( 705 vs. $722 \mathrm{~ms}$, for the additional object $=$ distractor and additional object $=$ target conditions, respectively) were statistically not significant, $F<1$.

The results quite clearly show that the additional object failed to capture attention. ${ }^{7}$ Experiment 3 was a replication

\footnotetext{
${ }^{7}$ A reviewer queried whether the elimination of the baseline trials, which would have made the additional object trials less salient, could have influenced results. Since the search set sizes used exceeded the subitizing limit ( $\sim 4$ objects), we do not think that observers would have been aware that the final array was larger than the initial array by 1. The results of a small control experiment to check whether observers could tell apart baseline (no additional object) and additional object trials when the search set was large showed that observers' performance was essentially at chance.
}

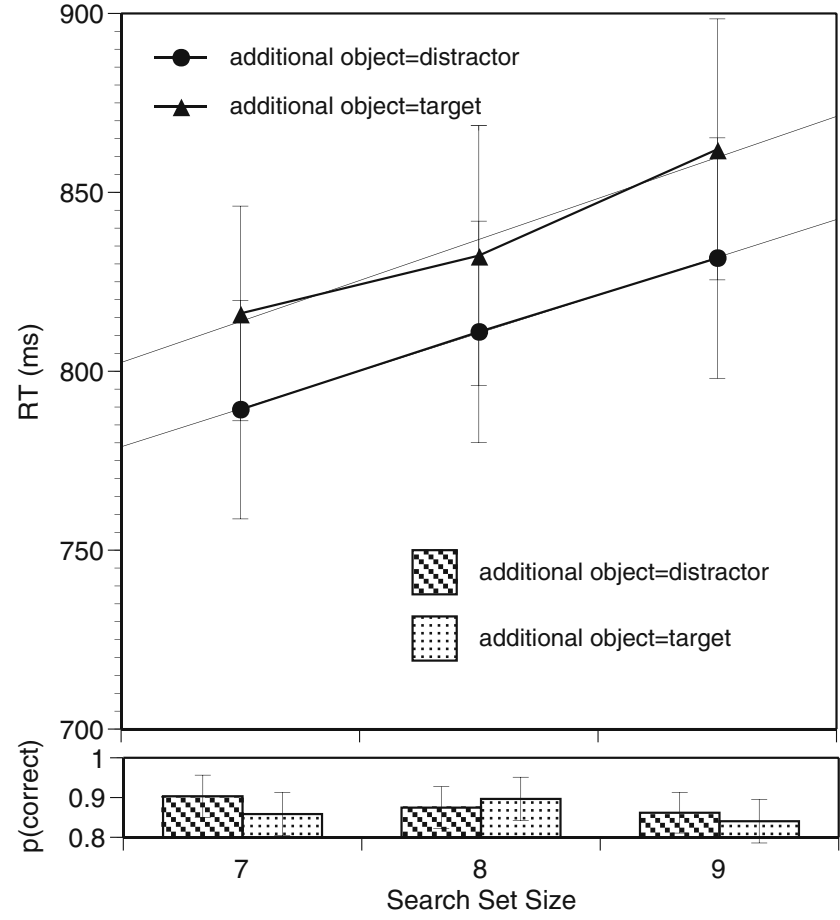

Fig. 5 Mean RTs of the two additional object conditions as a function of search set size. The best fitting linear regression functions for the various conditions are drawn. Error bars $=1 S E$

of Experiment 2a, with the search set size enlarged well beyond the capacity of VSTM. It is unlikely that the observers would be capable of tracking simultaneously the entire set of moving objects. When all the objects cannot be successfully tracked, the additional object cannot be automatically flagged, and, as a consequence, it fails to garner attentional prioritization.

\section{General discussion}

In the paradigm introduced by Franconeri et al. (2005), the objects that later turned into search letters remained stationary throughout the trial while an annulus contracted as the trial progressed. When the annulus concealed the objects midway through the trial, an additional object was inserted into the display under the annulus, thus rendering invisible the object's onset transients. Franconeri et al. showed that an object that was added in this fashion failed to capture attention. The additional object succeeded in capturing attention only when its transients were visible. They concluded that onset transients are critical for capture to occur. In Experiment 2, we used a variation of the Franconeri et al. paradigm: The annulus was kept stationary while the objects moved. At the crucial juncture, when the objects moved under the annulus, an additional object was 
added. As in Franconeri et al., all that the observers saw at this point was the annulus and nothing else. The results of Experiment 2 urge a different conclusion: Capture can occur without the presence of onset transients.

The findings of change blindness experiments show that, even when the display is encoded explicitly, our representation may not be necessarily veridical. Because VSTM has a limited capacity, a complex display will almost certainly not be completely represented (Simons, 1996; Simons \& Ambinder, 2005; Simons \& Levin, 1997). The displays in the Franconeri et al. (2005) were, however, quite austere, containing at the start of the trial only three or four or five identical objects. It is unlikely, therefore, that a complete representation of the display will overwhelm VSTM capacity. But, unlike a change blindness experiment, the search task did not require explicit encoding of the placeholders' locations. Neither is it apparent that implicit encoding could occur. Some commentators (O'Regan, 1992; O'Regan \& Nöe, 2001) have argued that no internal representation needs be maintained since the external world may be used as an extension of our memory.

In the Franconeri et al. (2005) procedure, the additional object-inserted under the annulus, thus making its onset transients invisible - can be distinguished from the initial set of objects only if the system solves the correspondence problem. This entails encoding the initial display, since only then can spatiotemporal continuity be established between the objects before their concealment and the search letters when they are revealed (Yantis \& Gibson, 1994). Once correspondence is established, one object will stand out as the only entity that cannot be reidentified (Pylyshyn, 2007). It will be flagged as new. But it is unclear whether the Franconeri et al. procedure facilitated encoding of the initial display.

Franconeri et al.'s (2005) results are interesting because they can be interpreted in two ways. First, the failure of observing attentional capture by a new object could imply that there was no representation of the initial display in the first place. On this view, the correspondence problem cannot be solved, and therefore the new object cannot be distinguished from the old objects. That is, it may be seen as a change blindness experiment in which the observer failed to detect a change. When the visual system has no capacity of identifying the additional object as a new entity, the question whether that object will capture attention becomes irrelevant. The second possibility is that implicit encoding obtained, and the visual scene was represented fully. Here, the visual system was fully capable of solving the correspondence problem and therefore of identifying the addition as a new entity. But the new object still did not receive prioritization. The failure to observe capture could be taken as evidence against the new object hypothesis. On this second view, capture ought not to be observed in Experiment 2. But the results showed that the new object had been prioritized.

There are two reasons why attentional capture by the new object was observed in Experiment 2. First, unlike in Franconeri et al. (2005), the annulus was kept stationary, which meant that it was unlikely to wrest attention away from the placeholders (cf. Davoli et al., 2007). Second, since the placeholders moved continuously, attention was likely to be focused on them (Abrams \& Christ, 2003; Franconeri \& Simons, 2003). Their representations in VSTM would be continuously updated as they moved across the display. Midway through a trial, the objects disappeared under the annulus. But because they were obscured only momentarily $(\sim 10 \mathrm{~ms})$, it is unlikely that their internal representations were lost. The objects then reappeared as letters. Because the objects had hitherto been continuously tracked, and their trajectories were entirely predictable, correspondence could easily be established between them and the search letters as they emerged from concealment. For trials in which an additional object was inserted during concealment, it would be the only object for which no spatiotemporal continuity could be established and was thus automatically flagged (Yantis \& Gibson, 1994).

If the correspondence problem can be solved only if the moving objects are effectively tracked, a new object should fail to capture attention when the system fails to track the entire set. This hypothesis was tested in Experiment 3. As in Experiment 2, the objects moved while the annulus remained stationary, thus ensuring that attention would be focused on the objects and not on the annulus. The set size of the moving objects was increased, exceeding the capacity limits revealed by the MOT experiments. Under these circumstances, the new object failed to capture attention. It is not enough to attend to the moving objects; what appears critical is that each individual object has to be encoded and its changing location updated. Only then will the visual system have the capacity of picking out the new object from among the old ones.

There was one perplexing result from these experiments. In Experiments $2 \mathrm{a}$ and 2b, the slope of the additional object $=$ target condition was flat, but the intercept was significantly higher than that in the additional object $=$ distractor condition. In those experiments, the observers were not forewarned that an additional object could be inserted to the display. When observers were adequately forewarned (Experiment 2c), the intercepts of the two additional object conditions were comparable. In Experiments $2 \mathrm{a}$ and $2 \mathrm{~b}$, the additional object $=$ target condition was relatively rare, which meant that it was unlikely that the observer would immediately engage the target when search commenced. As a result, the observer is likely to engage in postidentification checking to confirm that the letter was indeed the target 
before making the response. Forewarning the observers probably eliminated the postidentification checking. In general, the forewarned observers appear also to make faster responses. ${ }^{8}$ Expecting a change and seeing the change realized possibly shortened the overall decision latency. What is clear is that instructions to observers can influence considerably their task strategies. In this context, Hollingworth et al.'s (2010) failure to replicate the results of Davoli et al. (2007) is intriguing. We are currently working on experiments to unravel this mystery.

\section{References}

Abrams, R. A., \& Christ, S. E. (2003). Motion onsets capture attention. Psychological Science, 14, 427-432. doi:10.1111/ 1467-9280.01458

Becker, M. W., \& Pashler, H. (2002). Volatile visual representations: Failing to detect changes in recently processed information. Psychonomic Bulletin \& Review, 9, 744-750.

Breitmeyer, B., \& Ganz, L. (1976). Implications of sustained and transient channels for theories of visual pattern masking, saccadic suppression, and information processing. Psychological Review, 83, 1-36. doi:10.1037/0033-295X.83.1.1

Breitmeyer, B., \& Julesz, B. (1975). The role of on and off transients in determining the psychophysical spatial frequency response. Vision Research, 15, 411-415. doi:10.1016/0042-6989(75)90090-5

Chua, F. K. (2009). A new object captures attention-but only when you know it's new. Attention, Perception, \& Psychophysics, 71, 699-711. doi:10.3758/APP.71.4.699

Cowan, N. (2001). The magical number 4 in short-term memory: A reconsideration of mental storage capacity. The Behavioral and Brain Sciences, 24, 87-114. doi:10.1017/S0140525X01003922

Davoli, C. C., Suszko, J. W., \& Abrams, R. A. (2007). New objects can capture attention without a unique luminance transient. Psychonomic Bulletin \& Review, 14, 338-343.

Franconeri, S. L., Hollingworth, A., \& Simons, D. J. (2005). Do new objects capture attention? Psychological Science, 16, 275-281. doi:10.1111/j.0956-7976.2005.01528.x

Franconeri, S. L., \& Simons, D. J. (2003). Moving and looming stimuli capture attention. Perception \& Psychophysics, 65, 999-1010.

Gellatly, A., Cole, G., \& Blurton, A. (1999). Do equiluminant object onsets capture visual attention? Journal of Experimental Psychology: Human Perception and Performance, 25, 1609-1624. doi:10.1037/0096-1523.25.6.1609

Hollingworth, A., Simons, D. J., \& Franconeri, S. L. (2010). New objects do not capture attention without a sensory transient. Attention, Perception, \& Psychophysics, 72, 1298-1310. doi:10.3758/APP.72.5.1298

Jonides, J., \& Yantis, S. (1988). Uniqueness of abrupt visual onset in capturing attention. Perception \& Psychophysics, 43, 346-354.

Müller, H. J., \& Rabbitt, P. M. A. (1989). Reflexive and voluntary orienting of visual attention: Time course of activation and

\footnotetext{
${ }^{8}$ The slopes of the additional object $=$ distractor condition were comparable in all three experiments, $(35.3,32.2$, and 30.4 , for Experiments $2 \mathrm{a}, 2 \mathrm{~b}$, and $2 \mathrm{c}$, respectively). However, the intercepts were quite different. For Experiments $2 \mathrm{a}$ and $2 \mathrm{~b}$, in which the observers were not forewarned, the intercepts were 523 and $589 \mathrm{~ms}$, respectively. For Experiment 2c, the intercept was $446 \mathrm{~ms}$.
}

resistance to interruption. Journal of Experimental Psychology: Human Perception and Performance, 15, 315-330.

Nakayama, K., \& Mackeben, M. (1989). Sustained and transient components of focal visual attention. Vision Research, 29, 16311647. doi:10.1016/0042-6989(89)90144-2

O’Regan, J. K. (1992). Solving the "real" mysteries of visual perception: The world as an outside memory. Canadian Journal of Psychology, 46, 461-488.

O'Regan, J. K., \& Nöe, A. (2001). A sensorimotor account of vision and visual consciousness. The Behavioral and Brain Sciences, 24, 939-1031. doi:10.1017/S0140525X01000115

Pylyshyn, Z. W. (1989). The role of location indexes in spatial perception: A sketch of the FINST spatial-index model. Cognition, 32, 65-97. doi:10.1016/0010-0277(89)90014-0

Pylyshyn, Z. W. (2004). Some puzzling findings in multiple object tracking: I. Tracking without keeping track of object identities. Visual Cognition, 11, 801-822. doi:10.1080/13506280344000518

Pylyshyn, Z. W. (2007). Things and places: How the mind connects with the world. Cambridge: MIT Press.

Rauschenberger, R. (2003). When something old becomes something new: Spatiotemporal object continuity and attentional capture. Journal of Experimental Psychology: Human Perception and Performance, 29, 600-615. doi:10.1037/0096-1523.29.3.600

Rensink, R. A. (2000). Seeing, sensing, and scrutinizing. Vision Research, 40, 1469-1487. doi:10.1016/S0042-6989(00)00003-1

Simons, D. J. (1996). In sight, out of mind: When object representations fail. Psychological Science, 7, 301-305. doi:10.1111/ j.1467-9280.1996.tb00378.x

Simons, D. J., \& Ambinder, M. S. (2005). Change blindness: Theory and consequences. Current Directions in Psychological Science, 14, 44-48. doi:10.1111/j.0963-7214.2005.00332.x

Simons, D. J., \& Levin, D. T. (1997). Change blindness. Trends in Cognitive Sciences, 1, 261-267. doi:10.1016/S1364-6613(97) 01080-2

Theeuwes, J. (1995). Abrupt luminance change pops-out: Abrupt color change does not. Perception \& Psychophysics, 57, 637644.

Theeuwes, J. (2004). Top-down search strategies cannot override attentional capture. Psychonomic Bulletin \& Review, 11, 65-70.

Todd, J. J., \& Marois, R. (2004). Capacity limit of visual short-term memory in human posterior parietal cortex. Nature, 428, 751754. doi: $10.1038 /$ nature 02466

Todd, J. T., \& van Gelder, P. (1979). Implications of a transientsustained dichotomy for the measurement of human performance. Journal of Experimental Psychology: Human Perception and Performance, 5, 625-638.

Wolfe, J. M. (1999). Inattentional amnesia. In V. Coltheart (Ed.), Fleeting memories: Cognition of brief visual stimuli (pp. 71-94). Cambridge: MIT Press.

Yantis, S. (1998). Control of visual attention. In H. Pashler (Ed.), attention (pp. 223-256). London: Psychology Press.

Yantis, S. (2000). Goal-directed and stimulus-driven determinants of attentional control. In S. Monsell \& J. Driver (Eds.), Attention and Performance (Vol. 18, pp. 73-103). Cambridge, MA: MIT Press.

Yantis, S., \& Gibson, B. S. (1994). Object continuity in apparent motion and attention. Canadian Journal of Experimental Psychology, 48, 182-204. doi:10.1037/1196-1961.48.2.182

Yantis, S., \& Hillstrom, A. P. (1994). Stimulus-driven attentional capture: Evidence from equiluminant visual objects. Journal of Experimental Psychology: Human Perception and Performance, 20, 95-107. doi:10.1037//0096-1523.20.1.95

Yantis, S., \& Jonides, J. (1990). Abrupt visual onsets and selective attention: Voluntary versus automatic allocation. Journal of Experimental Psychology: Human Perception and Performance, 16, 121-134. doi:10.1037//0096-1523.16.1.121 